\title{
Hierarchical decision making for flood risk reduction
}

\author{
Custer, Rocco; Nishijima, Kazuyoshi
}

Published in:

Proceedings of the 11th International Conference on Structural Safety \& Reliability

Publication date:

2013

Link back to DTU Orbit

Citation (APA):

Custer, R., \& Nishijima, K. (2013). Hierarchical decision making for flood risk reduction. In Proceedings of the 11th International Conference on Structural Safety \& Reliability

\section{General rights}

Copyright and moral rights for the publications made accessible in the public portal are retained by the authors and/or other copyright owners and it is a condition of accessing publications that users recognise and abide by the legal requirements associated with these rights.

- Users may download and print one copy of any publication from the public portal for the purpose of private study or research.

- You may not further distribute the material or use it for any profit-making activity or commercial gain

- You may freely distribute the URL identifying the publication in the public portal

If you believe that this document breaches copyright please contact us providing details, and we will remove access to the work immediately and investigate your claim. 


\title{
Hierarchical decision making for flood risk reduction
}

\author{
R. Custer \& K. Nishijima \\ Technical University of Denmark, Lyngby, Denmark
}

\begin{abstract}
River flood events often cause large economic damages and casualties requiring stakeholders to manage flood risk. In flood prone areas, flood risk management can be achieved through a series hierarchically integrated protection structures, which together form a hierarchical flood protection system. In current practice, structures are often optimized individually without considering benefits of having a hierarchy of protection structures. It is here argued, that the joint consideration of hierarchically integrated protection structures is beneficial. A hierarchical decision model is utilized to analyze and compare the benefit of large upstream protection structures and local downstream protection structures in regard to epistemic uncertainty parameters. Results suggest that epistemic uncertainty influences the outcome of the decision model and that, depending on the magnitude of epistemic uncertainty the hierarchical level on which risk reducing measures are most beneficial might change.
\end{abstract}

\section{INTRODUCTION}

\subsection{Background}

River flood events cause large economic damages and casualties requiring public and private stakeholders to manage flood risk. Flood risk constantly changes due to amongst others, urbanization of previously uninhabited areas, deterioration of existing protection structures and climate change (Merz, et al. 2010; Novoa, 2013). As a consequence flood risk management strategies have to be continuously reevaluated and updated if necessary.

To cope with these challenges, decision makers have a plethora of possible risk reduction measures to choose from. These can be divided into structural measures, which physically restrain floodwaters (e.g. dams, dikes, local flood barriers, sand bags), and non-structural measures (e.g. early warning system). In this paper only structural measures are considered.

The nature of structural measures varies greatly in terms of cost, size of the protected area and time required for implementation etc. Large dams protect whole flood plains and often require over a decade from inception of planning to operation. On the other extreme, local flood barriers protect areas up to a neighborhood and require only few months for implementation.

In flood prone areas flood risk management is often achieved through a combination of hierarchically integrated defense structures, which together form a comprehensive flood protection system (Figure 1).

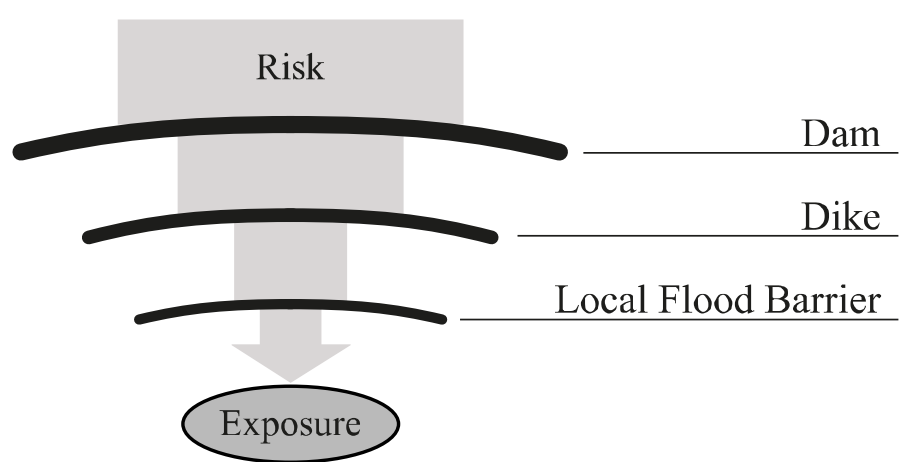

Figure 1. Schematic representation of hierarchical flood protection system.

The geographical reach of each protection structure is different. Figure 1 illustrates the hierarchical protection system from the perspective of an individual exposure, i.e. the exposure is protected by one structure on each hierarchical level.

When the whole river system is considered, a different picture is encountered, as each hierarchical level may entail a number of structures (see Figure 2).

The integration of several protection hierarchies can be permanent or temporal. Examples of situation where the integration is temporal include the construction of large-scale defense structure, where complementing measures at a lower hierarchical level might be needed while the main structure is under construction. 


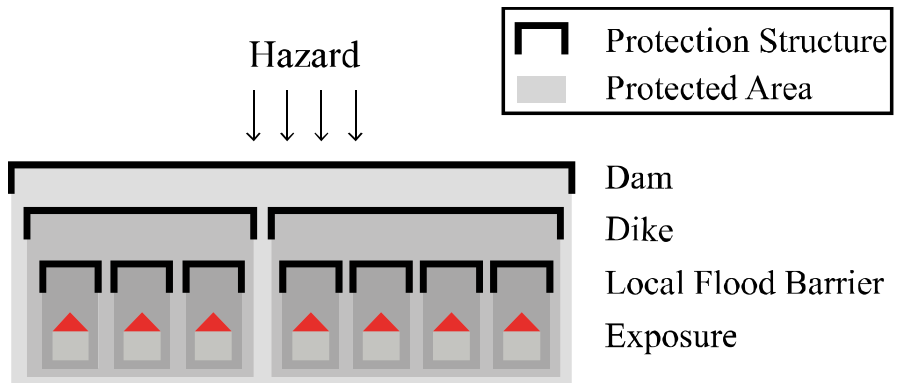

Figure 2. Hierarchical flood protection system.

A permanent protection hierarchy can be of use in situations where the planning of risk management strategy is complicated by uncertainty about the evolution of risk over time, e.g. due to climate change or urbanization of previously uninhabited areas. In this situation large structures, which need to be planned far in advance, can be complemented by smaller flexible structures which can be quickly implemented and updated if and when changing risk would require it. Temporal or permanent, in either situation decision makers are requested to identify the optimal flood protection system configuration for a river ba$\sin$.

In current practice, structures are often optimized individually, that is, without considering potential benefits of having several hierarchically integrated structures. It is here argued that it is beneficial to jointly consider all hierarchically integrated protection structures in a river system. The advantages of a joint evaluation of flood risk reduction measures are recognized in literature, see Dawson et al. 2004. and Nehlsen \& Wilke 2007.

\subsection{Hierarchical decision making}

Hierarchical decision making is here understood as a decision process which considers several hierarchically integrated components of a system. In contrast, single component decision making only considers one component at a time. In the context of flood protection systems, the components are protection structures.

Hierarchical decision making has been researched in several other topical areas, such as structural engineering (Vadde et al. 1994), mechanical engineering (Kuppuraju et al. 1985) and infrastructure management (Gómez et al. 2013). It is noted, that in these papers hierarchy has a different connotation as it refers to a system and hierarchically subordinated subsystems, i.e. components, whereas in the present paper hierarchy refers to a number of components which are hierarchically integrated.

In the context of flood protection system, hierarchical decision making presents several benefits in comparison to single structure decision making, as it allows approaching questions that can otherwise not be answered. In the following four instances are described, where the utilization of a hierarchical consideration is beneficial.
1) When a decision maker is responsible for only one protection structure, it allows identifying the optimal decision alternative for the structure in his responsibility under consideration of all other structures in the protection system. The decision maker may also consider possible future changes on other hierarchical levels of the protection system, and optimized its structure accordingly. An example of such a situation is a company that has to decide how to best protect its facilities from floods under consideration of the government's present and future flood risk reducing actions.

2) Decision makers have to identify on which hierarchical level risk reduction measures are most beneficial. This situation can be encountered e.g. when central government has to decide to which local entity to allocate money for flood risk management.

3) Decision makers want to identify the optimal configuration of the flood protection system, which is found through a joint optimization of all hierarchically integrated protection structures.

4) Decision makers want to consider, and if necessary plan, risk reduction capacity in a flood protection system. As previously mentioned, such a situation is encountered when large protection structures are constructed but the future evolution of flood risk is uncertain.

\subsection{Challenges}

Little research is found in regard to hierarchical decision making in the context of flood risk management. Whereas the necessity of a joint consideration of flood risk protection structure is acknowledged in Dawson et al. 2004, the challenges for solving such a decision problem are not found in literature. In the following three challenges are identified and described.

Firstly, flood risk reduction decision making generally involves hydrodynamic modeling which is computationally expensive. Each alternative flood protection system configuration needs to be considered separately in the hydrodynamic model. Moreover, the accurate evaluation of flood risk may require the modeling of breaching scenario for each prospective protection structure, see Dawson et al. 2005 , bringing the required number of runs of the hydrodynamic model to prohibitive levels. As a consequence an efficient modeling framework is required which allows reducing the computational costs of hydrodynamic modeling.

A second challenge is identified in dealing with probabilistic dependencies at different hierarchical levels. In particular, when flood damages are aggregated from a low spatial scale to a higher scale it has to be considered that damage distribution are dependent, e.g. on epistemic uncertainty parameter, 
and can thus not be simply convoluted. Although a well known problem, a computationally efficient solution is not readily at hand.

Lastly, it is important to identify in which situations the hierarchical consideration is particularly relevant. It is mathematically trivial that hierarchical consideration, when compared to single structure consideration, generally leads to lower costs and thus more beneficial system configuration. Nevertheless, the added benefit of utilizing the hierarchical consideration may vary depending on the analyzed system and its uncertainties. In particular, the benefit of hierarchical decision model might depend on the epistemic uncertainty of hazard, the epistemic uncertainty of the spatial distribution on exposure, the degree of spatial concentration of the exposure and to the uncertainty in exposure's vulnerability.

\subsection{Paper outline}

This paper takes on the third challenge. By considering all hierarchically integrated structures in a protection system it aims at identifying for which uncertainty parameterizations, large upstream protection structures are more beneficial than local downstream protection structures and vice versa. In this regard, several hypotheses are formulated and tested through a sensitivity analysis in respect to several uncertainty parameters.

The structure of the paper is as follows. First, a decision model for hierarchical decision making is introduced. Thereafter an example is presented and several of its parameters are varied in the aforementioned sensitivity analysis. Further the results are presented and discussed, followed by conclusions.

\section{METHODOLOGY}

A decision model for hierarchical decision making in the context of flood risk reduction is introduced.

\subsection{Decision alternatives}

A system with $L$ hierarchically integrated protection levels is considered. The decision alternatives at the $l$-th hierarchical level, $\quad l=1,2, \ldots, L, \quad$ are $A_{l}=\left\{a_{l}^{(0)}, a_{l}^{(1)}, \ldots, a_{l}^{(m)}\right\}$, where $a_{l}^{(0)}$ identifies the "no action" alternative, i.e. the status quo.

Each protection level might include a number of structures which operate in parallel and can be implemented independently from each other (as illustrated in Figure 2). Note that decision alternatives do not represent the state of an individual structure, but rather all mutually exclusive combinations of states of all structures on a hierarchical level. For instance, if hierarchical level $l$ includes $s$ individual structures and each individual structure can be in two mu- tually exclusive states, $A_{l}$ will include $m=2^{s}$ decision alternatives.

A decision alternative for the whole protection system is represented by $\mathbf{a}^{(\cdot)}$ which combine a decision alternative for each hierarchical level as $\mathbf{a}^{\left(i_{1}, i_{2}, \ldots, i_{L}\right)}=\left(a_{1}^{\left(i_{1}\right)}, a_{2}^{\left(i_{2}\right)}, \ldots, a_{L}^{\left(i_{L}\right)}\right)$. The set of different decision alternatives for the whole protection system is $A=\left\{\mathbf{a}^{\left(i_{1}, i_{2}, \ldots, i_{L}\right)} \forall i_{1}, i_{2}, \ldots, i_{L}\right\}$. Depending on the decision problem, constraints might be introduced on $A$, e.g. if the decision maker only have control over one hierarchical level.

\subsection{Decision model}

The vector $\mathbf{X}=\left(X_{1}, X_{2}, \ldots, X_{N}\right)$ contains all random variable influencing the decision problem. Moreover all other parameters influencing the decision, which are not considered random, are collected in vector $\boldsymbol{\gamma}=\left(\gamma_{1}, \gamma_{2}, \ldots, \gamma_{n}\right)$.

The cost of decision alternative $\mathbf{a}^{\left(i_{1}, i_{2}, \ldots, i_{L}\right)}$ are modeled as $C\left(\mathbf{a}^{\left(i_{1}, i_{2}, \ldots, i_{L}\right)}, \boldsymbol{\gamma}, \mathbf{X}\right)$. In general $C(\cdot)$ includes the net present value of construction costs and the flood damages over the lifetime of the structure. The expected costs $c\left(\mathbf{a}^{\left(i_{1}, i_{2}, \ldots, i_{L}\right)}, \boldsymbol{\gamma}\right)$ are calculated as

$c\left(\mathbf{a}^{\left(i_{1}, i_{2}, \ldots, i_{L}\right)}, \boldsymbol{\gamma}\right)=E_{\mathbf{X}}\left[C\left(\mathbf{a}^{\left(i_{1}, i_{2}, \ldots, i_{L}\right)}, \boldsymbol{\gamma}, \mathbf{X}\right)\right]$.

The optimal system configurations $\mathbf{a}^{*} \in A$ minimizes costs from Equation (1), and is found as

$$
c^{*}(\boldsymbol{\gamma})=c\left(\mathbf{a}^{*}, \boldsymbol{\gamma}\right)=\min _{A} E_{\mathbf{X}}\left[C\left(\mathbf{a}^{\left(i_{1}, i_{2}, \ldots, i_{L}\right)}, \boldsymbol{\gamma}, \mathbf{X}\right)\right] .
$$

\section{SENSITIVITY ANALYSIS}

The purpose of the sensitivity analysis introduced in the following is to assess in which situations the hierarchical optimization is most beneficial, i.e. test the hypotheses introduced at the end of this section.

\subsection{Model setup}

\subsubsection{Introduction}

An example is developed for an area in Switzerland. Whereas a real digital elevation model (DEM) and course of the river Aar are utilized, the hazard, exposure and the nature of protection structures are postulated.

The study area is divided into grid cells with a resolution of $200 \mathrm{~m}$; hazard and exposure are calculated respectively distributed at a grid cell level. A flood protection system with two hierarchically integrated protection structures is considered. On the upstream hierarchical level a dike is considered whereas on the downstream hierarchical level local flood barriers (LFB, see e.g. Bramley \& Bowker 2002) are considered. The portfolio of exposure, i.e. 
the property that might get affected by floods, is distributed over the study area. A schematic representation of the study area is given in Figure 3.

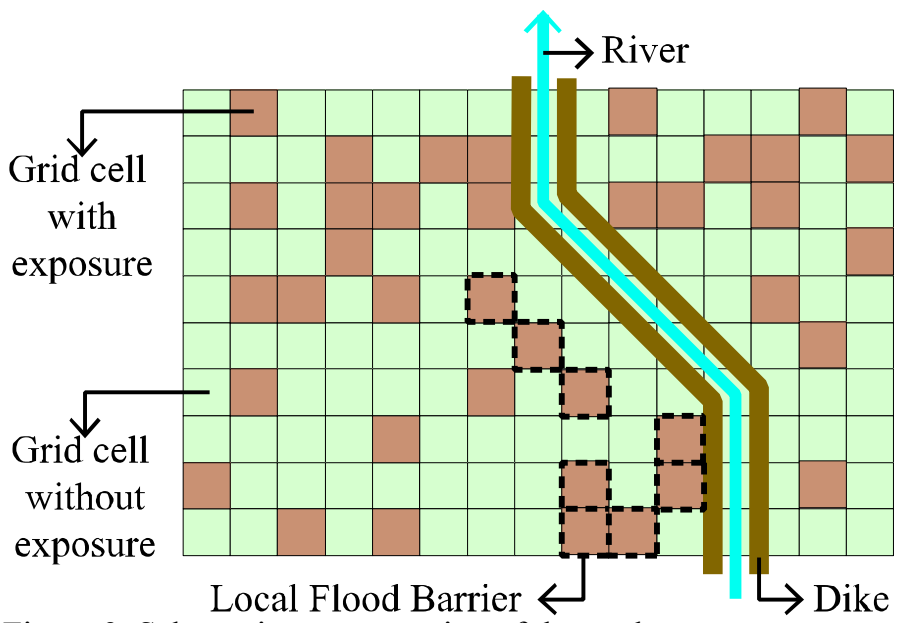

Figure 3. Schematic representation of the study area.

In the example the future evolution of climate is considered a major source of hazard uncertainty. Two climate scenarios are postulated and it is assumed that a decision in regard to either protection structure can be taken at present time or at one time in the future when further information on climatic evolution is available.

To allow general conclusions to be drawn, a large number of different parameterizations of the example model, i.e. combinations of parameters in vector $\gamma$, are considered and the average tendencies are investigated.

\subsubsection{Upstream hierarchical level}

It is assumed that a dike with $5 \mathrm{~m}$ height already exists and the decision makers have the choice to leave the dike unchanged (decision alternative $a_{1}^{(0)}$ ) or upgrade the dike's height to $7 \mathrm{~m}$ (decision alternative $\left.a_{1}^{(1)}\right)$. The construction costs are postulated as $c c_{1}^{(0)}=0$ for $a_{1}^{(0)}$ and $c c_{1}^{(1)}=10^{8} \mathrm{CHF}$ for $a_{1}^{(1)}$. If the dike is upgraded the construction time is assumed to be $t_{c}=10$ years and the lifetime is assumed to be $T=100$ years. The area protected by the dike comprises the whole flood plain and thus all exposures.

\subsubsection{Downstream hierarchical level}

The decision maker has the possibility to install local flood barriers to complement the dike. It is considered that a LFB protects one grid cell and as such, an independent decision is taken for each grid cell. Thus, the number of decision alternatives for the downstream hierarchical level is $2^{K}$ where $K$ is the number of grid cells. The protection height of an LFB is $h_{L F B}=1.5 \mathrm{~m}$ and the construction costs for a single LFB are $c c_{L F B}=10^{6} \mathrm{CHF}$. As for the dike, the lifetime of LFBs is $T=100$ years; the construction period is disregarded as it is comparatively short.

\subsubsection{Exposure portfolio}

The spatial distribution of exposure in the portfolio has a large impact on the result of the decision model. To allow drawing general conclusions, the influence of a particular spatial distribution on the results should be minimized. For this reason, for each model run a different exposure distribution is considered and the average tendency investigated. The different realizations of exposure distribution are created according to the parameterization and methodology introduced in the following.

The total value of exposure, $v_{t o t}$, is postulated and distributed to a certain number, $K_{P T F}$, of grid cells. The number of grid cells with exposure is modeled as $K_{P T F}=\gamma_{d} \cdot K$, where $K$ is the total number of grid cells. Thus parameter $\gamma_{d}$ controls the degree of spatial dispersion of exposure. The exposure value attributed to the $k$-th grid cell is $V_{k}$, the value attributed to grid cells with exposure is $v_{k}=v_{t o t} / n_{P T F}$, all other grid cells will have no exposure value on them, thus $v_{k}=0$. A realization of exposure distribution is $\omega=\left(v_{k}, k=1, \ldots, K\right)$. Note that in each realization of exposure distribution the grid cells to which the exposure is allocated are randomly selected.

In many situations the decision maker's knowledge about the exact location of exposures is lacking; to consider the degree of knowledge of the decision maker, the parameter $\gamma_{u}>1 \in \mathbf{N}$ is introduced; it stands for the number of equally likely exposure distribution considered in the decision model. As such, if $\gamma_{u}=1$ only one realization of exposure distribution is considered, i.e. the knowledge about the portfolio is perfect. On the other hand, if $\gamma_{u}=\eta$ and $\eta$ is large knowledge about the spatial distribution of exposure is very limited and uncertainty high. The optimal decision is found under consideration of $\eta$ different spatial distributions of exposure. Each of the $\gamma_{u}$ exposure distributions considered in one decision problem have the same $v_{t o t}$ and $\gamma_{d}$, the difference lays in which grid cells carry the exposure. As mentioned these are randomly selected for each exposure distribution.

\subsubsection{Digital elevation model}

The digital elevation model DHM25/200 from the Swiss Federal Office of Topography, swisstopo, is utilized; its resolution is $200 \mathrm{~m}$. Digital elevation model are associated with an inherent uncertainty, from measurement errors as well as from the elevation distribution within a grid cell. The latter is considered through the standard deviation $\sigma_{D E M}$. For each grid cell $k$, an uncertainty term $\Delta_{D E M, k} \sim N\left(0, \sigma_{D E M}\right)$ is added to the elevation given in the DEM, $h_{D E M, k}$, such that $H_{D E M, k}=h_{D E M, k}+\Delta_{D E M, k}$. The term $\Delta_{D E M, k}$ is modeled independently for each grid cell. 


\subsubsection{Hazard}

First the modeling of flood events is described; thereafter considerations on climate change and its parameterization are introduced.

Flood events $E=e_{j}, j=1, \ldots, J$ are considered in the example. The model considers the annual most critical flood space which is divided into $J=9$ flood events. For each event the river discharge time series is postulated at the upstream boundary of the study area. The shape of the discharge time series is assumed to be the same for each event, with a constant base flow of $Q_{\text {base }}=100 \mathrm{~m}^{3} / \mathrm{s}$ and a triangular peak as illustrate in Figure 4.

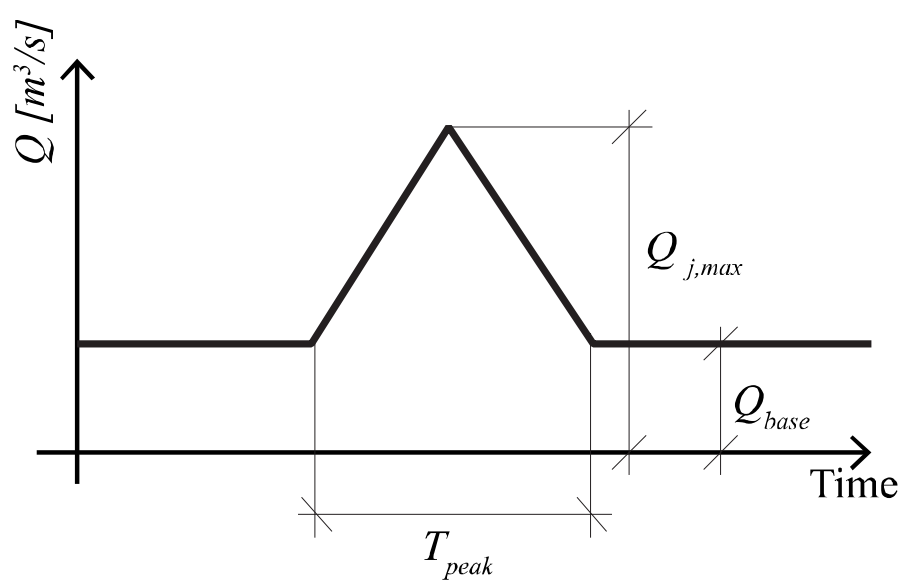

Figure 4. Schematic representation of the modeled discharge time series.

Whereas time $T_{\text {peak }}=200^{\prime} 000 \mathrm{~s}$, peak discharges $Q_{j, \max }$ vary for each event $e_{j}$, as follows

$Q_{j, \max }=j \cdot 100 \mathrm{~m}^{3} / \mathrm{s}$

The yearly probability, $P\left[E=e_{j}\right]$, of occurrence of event $e_{j}$ is modeled according to Equation (4).

$P\left[E=e_{j}\right]=\frac{\lambda^{j}}{j !} \exp (-\lambda), j=1, \ldots, 9$.

The river discharge serves as input into the hydrodynamic model, LISFLOOD-FP (P. D. Bates \& De Roo 2000), which models river runoff as well as flood plain inundation. The hydrodynamic model is run for each combination of event $e_{j}$ and decision alternative $a_{1}^{\left(i_{1}\right)}$ on the upstream hierarchical level. From each model run a hazard map is obtained, i.e. the inundation depth $w_{h, k}\left(a_{1}^{\left(i_{1}\right)}, e_{j}\right)$ for each grid cell $k$ in the study area.

Note that the structures at downstream hierarchical level, i.e. LFBs, are not included in the hydrodynamic model, but are considered through simple manipulation of hazard maps. The water depth, $w_{h, L F B, k}$, when an LFB is installed is found as follows:

$$
\left\{\begin{array}{l}
w_{h, L F B, k}=0, w_{h, k}<h_{L F B} \\
w_{h, L F B, k}=w_{h, k}, w_{h, k} \geq h_{L F B} .
\end{array}\right.
$$

This is justifiable as it is thought that LFB have a limited impact on the large scale hydrodynamic flows and can thus be omitted from the hydrodynamic model.

The uncertainty term of the DEM is considered in a similar manner. Small changes in the DEM are expected to translate directly into the same change in the inundation depth, therefore the uncertainty term is subtracted directly from the inundation depth yielded by the hydrodynamic model. Thus for grid cell $k$ the inundation depth will be: $W_{h, k}=w_{h, k}-\Delta_{D E M, k}$. The inundation depth with an LFB installed, $W_{h, L F B, k}$, is calculated analogously.

In the following the focus is shifted to the consideration of climate change. As previously mentioned, the future evolution of climate is considered as a source of hazard uncertainty. In this example two possible climates are modeled, namely $\Theta=\theta_{1}$ as the base scenario with constant climate and $\Theta=\theta_{2}$ as a scenario with climate change. To impact of the climate scenario on hazard is considered through parameter $\lambda$ in equation (4) which takes values $\lambda\left(\Theta=\theta_{1}\right)=0.2$ and $\lambda\left(\Theta=\theta_{2}\right)=1$. Furthermore it is modeled that a decision in regard to either structure is taken at present time $t=t_{0}$ under consideration that the decision regarding either structure can be delayed to one point in the future $\left(t=t_{1}\right)$. In this example $t_{1}=25$ is postulated.

At time $t=t_{0}$ the prior degree of belief of the current climate is $P\left[\theta_{1}\right]$. By time $t=t_{1}, P\left[\theta_{1}\right]$ can be updated with new evidence, aggregated in the indicator $Z_{\Theta}$, which can take values and $Z_{\Theta}=\left\{\theta_{1}, \theta_{2}\right\}$. The likelihoods of each climate scenario given indicator are postulated as: $P\left[Z_{\Theta}=\theta_{1} \mid \Theta=\theta_{1}\right]=0.9$ and $P\left[Z_{\Theta}=\theta_{2} \mid \Theta=\theta_{2}\right]=0.9$.

\subsubsection{Vulnerability}

The vulnerability model expresses the damage degree $D_{k}$, i.e. the damage in grid cell $k$ as a ratio of exposure value $V_{k}$, in function of the inundation depth with a logistic function as per Equation (6)

$D_{k}=\frac{\mathrm{e}^{\varphi}}{1+e^{\varphi}}$,

where $\varphi=a \cdot W_{h, k}+b$ and $a=1, b=-6 . W_{h, k}$ is straightforwardly substituted with $W_{h, L F B, k}$ when a LFB is installed in the grid cell.

For event $e_{j}$ and decision alternative $\mathbf{a}^{(\cdot)}$ the flood loss, i.e. damage in monetary terms, given exposure distribution $\omega$ is

$L\left(e_{j}, \mathbf{a}^{(\cdot)}, \omega\right)=\sum_{k=1}^{K} v_{k} D_{k}$.

\subsubsection{Costs of decision alternative}

In this example the vector $\gamma$ contains the following elements: $\gamma=\left(\sigma_{D E M}, \gamma_{u}, \gamma_{d}, P\left[\theta_{1}\right], v_{t o t}\right)$. The vector $\mathbf{X}$ contains the elements $\mathbf{X}=\left(\boldsymbol{\Delta}_{D E M}, \mathbf{V}, E\right)$ where $\boldsymbol{\Delta}_{D E M}$ 
is the vector containing the uncertainty term of the digital elevation model $\Delta_{D E M, k}, k=1, \ldots, K, \quad \mathbf{V}$ is a vector containing the exposure value $V_{k}, k=1, \ldots, K$ for each grid cell.

Yearly expected costs $c_{t}\left(\mathbf{a}^{(\cdot)}, \boldsymbol{\gamma}\right)$ for decision alternative $\mathbf{a}^{(\cdot)}$ are calculated according to Equation (1) under consideration of construction costs entailed by $\mathbf{a}^{(\cdot)}$ and the results of Equation (4) and Equation (7). Thereafter, the expected life time costs $c\left(\mathbf{a}^{(\cdot)}, \boldsymbol{\gamma}\right)$ for decision alternative $\mathbf{a}^{(\cdot)}$ are calculated by summing the net present value of yearly expected costs over $T=100$ years, i.e.

$c\left(\mathbf{a}^{(\cdot)}, \boldsymbol{\gamma}\right)=\sum_{t=1}^{T} c_{t}\left(\mathbf{a}^{(\cdot)}, \boldsymbol{\gamma}\right) \cdot r(t)$.

where $r(t)=\left(1+i_{r}\right)^{-\left(t-t_{0}\right)}$ is a discounting function, $i_{r}$ is the discounting rate, which is postulated to be $i_{r}=4 \%$, and $t_{0}$ is present time.

When calculating the costs of a decision alternative it has to be considered that it might entail different protection system configuration over time, e.g. during construction time of the main structure, or if it is decided to modify the protection structure at time $t=t_{1}$.

\subsection{Parameterization}

The results presented in the next section are based on $n_{r}=50^{\prime} 000$ model runs. In each model run the values of the parameters in vector $\gamma$ are varied. Also, realizations of $\mathbf{X}$ are randomly generated for each model run. Note that whereas the decision model is run for each realization of $\gamma$, this is not the case for each realization of $\mathbf{X}$ because these are random variables as per Equation (2).

\subsection{Hypotheses}

The hypotheses tested in the sensitivity analyses are listed in the following.

\subsubsection{Hypothesis 1}

The first hypothesis is that upstream structures are more beneficial when the uncertainty about the spatial distribution of the portfolio is large.

Here, the decision space $A$ is constrained such that only one structure is optimized at a time. The hypothesis is tested by comparing $c_{u p}(\gamma)$ and $c_{\text {down }}(\gamma)$ i.e. to expected costs when flood risk reduction measures are implemented on the upstream hierarchical level and on the downstream hierarchical level respectively.

\subsubsection{Hypothesis 2}

The second hypothesis states that upstream structures are more beneficial when the dispersion of the portfolio is large. The testing of this hypothesis is analogous to hypothesis 1 .

\subsubsection{Hypothesis 3}

The third hypothesis states that downstream structures are more beneficial when DEM uncertainty is large. The testing of this hypothesis is analogous to hypothesis 1 .

\section{RESULTS}

In the following the model results are presented for the formulated hypotheses. The results of model run $i=1, \ldots, n_{r}$ comprise the minimized costs $c_{u p}^{(i)}(\boldsymbol{\gamma})$ and $c_{\text {down }}^{(i)}(\gamma)$ which, when compared, allow to test the hypotheses formulated in the previous section. The comparison is facilitated by calculating ratio $r_{c}\left[\gamma_{s, 1}, \gamma_{s, 2}\right]$ which expresses the ratio between the number of model runs yielding $c_{u p}^{(i)}<c_{d o w n}^{(i)}$ and the total number of runs given $\gamma_{s, 1}<\gamma_{s}^{(i)^{(i)}}<\gamma_{s, 2}$

$$
r_{c}\left[\gamma_{s, l}, \gamma_{s, 2}\right]=\frac{\sum_{i=1}^{n_{r}} I\left[c_{u p}^{(i)}<c_{d o w n}^{(i)} \mid \gamma_{s, 1}<\gamma_{s}^{(i)}<\gamma_{s, 2}\right]}{\sum_{i=1}^{n_{r}} I\left[\gamma_{s, I}<\gamma_{s}^{(i)}<\gamma_{s, 2}\right]},
$$

where $I[\cdot]$ is an indicator function, which returns 1 when the statement in brackets is true and 0 otherwise, $\gamma_{s}$ is the element of $\gamma$ which is considered in the sensitivity analysis and $\gamma_{s, 1}, \gamma_{s, 2}$ are two values delimiting a bin.

Figure 5 illustrates the results of the sensitivity analysis in regard to $\gamma_{u}$, by plotting $\gamma_{s}=\gamma_{u}$ against $r_{c}$.

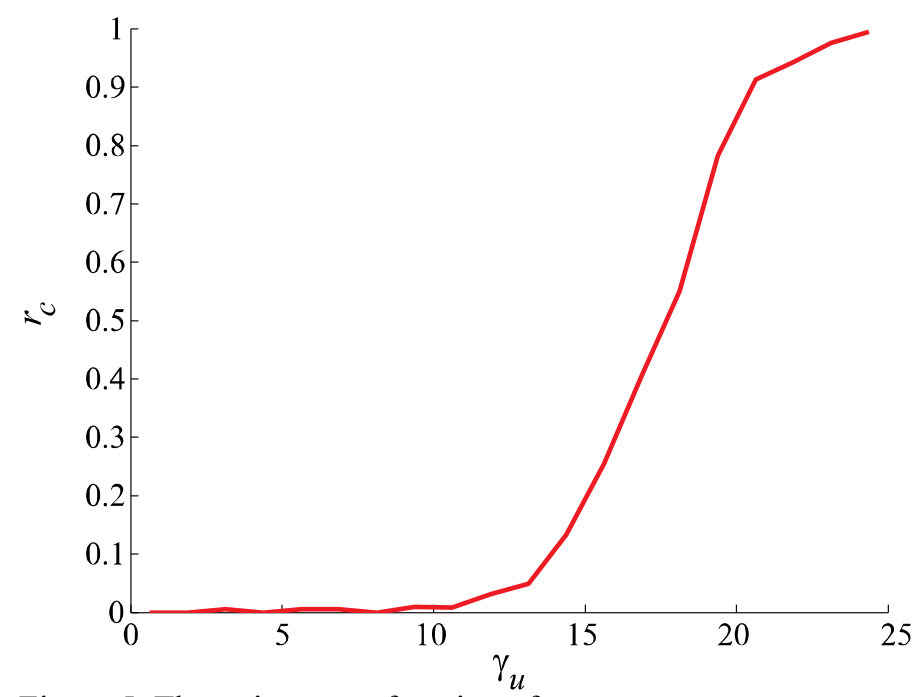

Figure 5. The ratio $r_{c}$ as a function of parameter $\gamma_{u}$.

Similarly, Figure 6 and Figure 7 illustrate the ratio $r_{c}$ for $\gamma_{s}=\sigma_{D E M}$ and $\gamma_{s}=\gamma_{d}$ respectively. 


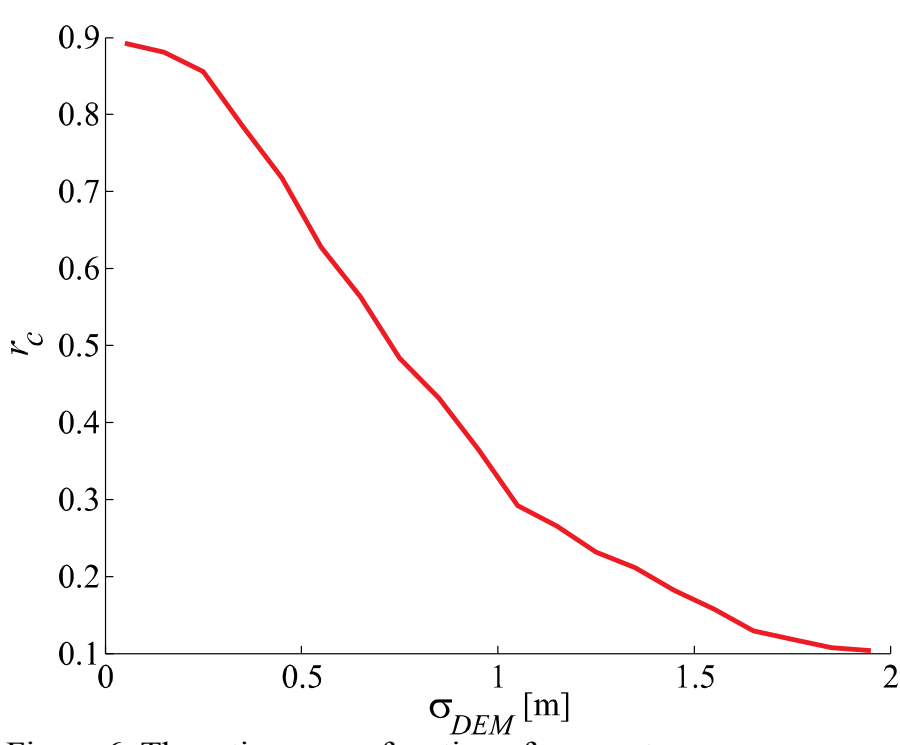

Figure 6. The ratio $r_{c}$ as a function of parameter $\sigma_{D E M}$.

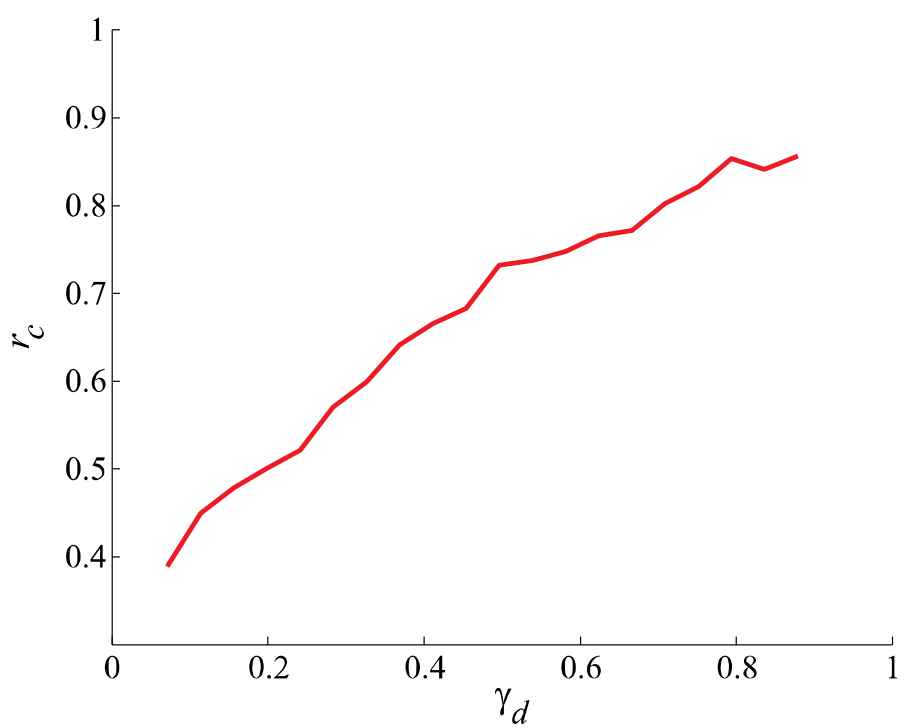

Figure 7. The ratio $r_{c}$ as a function of parameter $\gamma_{d}$.

\section{DISCUSSION}

In the following results are discussed in the light of the hypotheses formulated in Section 3.

A lack of knowledge in regard to the spatial distribution of exposure, i.e. a large value for $\gamma_{u}$, a dike is more beneficial than the installation of LFBs (Figure 5). This reckoning is relevant e.g. in developing countries where the location of exposure is often unknown.

Similar observations are made in regard to the spatial dispersion of the portfolio (Figure 6). When dispersion is large, a dike becomes more beneficial compared to LFBs. The reason for this is found to be that concentrated portfolios present higher exposure value per grid cell and thus the relatively cheap protection offered by LFBs is very effective whereas in dispersed portfolios, exposure value per cell is lower and expected damages for the cell cannot justify the expense for the construction of a LFB.

The analysis in regard to $\sigma_{D E M}$ (Figure 7) confirms the third hypothesis as it indicates downstream structures, i.e. LFB, are more beneficial then upstream structures when $\sigma_{D E M}$ is large. The reason is found to be that the benefit of LFB is mostly unaffected by an increase in uncertainty in the DEM, whereas the benefit of the dike decreases. LFB are unaffected by DEM uncertainty because they are installed locally, i.e. at the same geographical scale as DEM uncertainty is modeled. LFB are simply built on top of the uncertain terrain and thus offer protection up to $h_{L F B}$ over the terrain irrespective of $\Delta_{D E M}$.

Similar analyses are also undertaken in regard to $P\left[\theta_{1}\right]$. Results do not indicate any sensitivity in regard to $P\left[\theta_{1}\right]$.

The results confirm that epistemic uncertainty impact the result of hierarchical decision making. In particular the hierarchical level on which risk reducing measures are most beneficial may change depending on the magnitude of epistemic uncertainty. One implication of this finding is that a better understanding of the system, e.g. through collection of data, can lead to a completely different protection system.

Many aspects of the here presented example model are simplified, particularly the flood risk model underlying the decision model. In further studies a more sophisticated flood risk model should be utilized, for instance considering the probability of breaching of protection structures. Such consideration will require solving the challenge related to large computation time of hydrodynamic model mentioned in the introduction. Nevertheless it is noted that the presented model can be readily utilized in practical cases to identify on which hierarchical level flood risk reduction measures are most beneficial.

Further, efforts should be undertaken to extend the model to more hierarchical levels and to more decision alternatives per structure. Also, the models behavior with other hazard and orographic conditions should be tested.

Currently it is considered that the decisions can only be taken at times $t=t_{0}$ and $t=t_{1}$, in reality the decision can be taken at any time when new evidence becomes available. A methodology to extend the decision model to more time steps can be found in Nishijima \& Anders 2012.

\section{CONCLUSION}

In many situations flood risk is reduced through a combination of several risk reduction measures. In the case of structural measures, these might be hierarchically integrated to form a comprehensive flood protection system. Nevertheless, in current practice protection structures are often evaluated and optimized individually without considering the possibility of having several hierarchically integrated protection structures. In contrast, it is here argued that it 
is beneficial to jointly consider all hierarchically integrated protection structures in a river system. The situations, in which hierarchical consideration might be helpful, are given in the introduction alongside several challenges encountered in hierarchical decision making.

Hierarchical decision model allows approaching questions which could not be approached in a single structure decision model. For instance it allows decision makers to identify on which hierarchical level protection measures are most beneficial, e.g. to allocate money accordingly. For such a situation a sensitivity analysis is carried out. In particular it is analyzed what influence the epistemic uncertainty has on the benefit of large upstream structures (e.g. dike) versus local downstream protection structures (e.g. local flood barrier). Moreover, the same analysis is carried out in regard to the degree of dispersion of the portfolio.

The analysis shows that benefit of structures may vary depending on the magnitude of epistemic uncertainty. Although it cannot be concluded that large epistemic uncertainty automatically favors large upstream protection structures, the findings are quite clear. When exposure is spatially dispersed and its spatial distribution uncertain, a dike is generally more favorable then local flood barriers, whereas concentrated exposure with a well-known spatial distribution favors local flood barriers. Uncertainty in the digital elevation model renders large upstream protection structures less attractive, and thus favors local flood barriers.

\section{REFERENCES}

Bates, P.D. \& De Roo, A.P.J., 2000. A simple raster-based model for flood inundation simulation. Journal of Hydrology, 236(1-2), pp.54-77.

Bramley, M.E. \& Bowker, P.M., 2002. Improving local flood protection to property. Proceedings of the ICE - Civil Engineering, 150(5), pp.49-55.

Dawson, R, Hall, J \& Davis, J., 2004. Performance-based management of flood defence systems. , (March), pp.3544.

Dawson, Richard et al., 2005. Sampling-based flood risk analysis for fluvial dike systems. Stochastic Environmental Research and Risk Assessment, 19(6), pp.388-402.

Gómez, C. et al., 2013. Hierarchical infrastructure network representation methods for risk-based decision-making. ... and Infrastructure ..., (April 2013), pp.37-41.

Hall, J.W. et al., 2006. Impacts of climate change on coastal flood risk in England and Wales: 2030-2100. Philosophical transactions. Series A, Mathematical, physical, and engineering sciences, 364(1841), pp.1027-49.

Kuppuraju, N. et al., 1985. Hierarchical decision making in system design. Engineering Optimization, (April 2013), pp.37-41.

Merz, B. et al., 2010. Fluvial flood risk management in a changing world. Nat Hazards Earth Syst Sci, pp.509-527.

Nehlsen, E. \& Wilke, M., 2007. Flood Mitigation Using Cascading Dike System. In 8. Forum DKKV/CEDIM: Disaster Reduction in Climate Change.
Nishijima, K. \& Anders, A., 2012. Optimization of sequential decisions by least squares Monte Carlo method. In IFIP WG 7.5 Reliability and Optimization of Strucutral Systems. Jerevan, Armenia [Accepted for publication].

Novoa, D.C., 2013. The Economics of Climate Change, Urbanisation, and Long-term Flood Protection. In W. Leal Filho, ed. Climate Change and Disaster Risk Management. Berlin, Heidelberg: Springer Berlin Heidelberg, pp. 483496.

Vadde, S., Allen, J.K. \& Mistree, F., 1994. Compromise decision support problems for hierarchical design involving uncertainty. Computers \& Structures, 52(4). 